\title{
Longitudinal Hippocampal and Fornix Changes after Traumatic Brain Injury: Observations from Traditional Structural Magnetic Resonance Imaging
}

\author{
Jerome J Maller ${ }^{1 *}$ and Chantal Reglade-Meslin² \\ ${ }^{1}$ Monash Alfred Psychiatry Research Centre, The Alfred \& Monash University Central Clinical School, Melbourne Victoria, Australia \\ ${ }^{2}$ Centre for Mental Health Research, The Australian National University, Canberra, Australia
}

\begin{abstract}
Mild to moderate traumatic brain injury (TBI) often results in diffuse axonal injury and post-traumatic amnesia, with short- and long-term cognitive and neurological impairment. Whilst there are a variety of neuroimaging-based computerized techniques by which to investigate longitudinal brain changes after brain insult, there are some which can be appreciated by the naked eye from traditional T1-weighted structural magnetic resonance (MR) imaging without the need for further image manipulation or analysis. In this article we review such regions with a focus on the hippocampus and fornix based on longitudinal structural MR images from patients who have sustained a mild to moderate TBI.
\end{abstract}

Keywords: Magnetic resonance imaging; Traumatic brain injury; Hippocampus; Sulcal cavities; Fornix

\section{Overview}

TBI results from an external force applied to the cranium which may cause brain movement within this vault, often in a linear and/or rotational acceleration/deceleration manner with coup and contrecoup injury. A common outcome of this rapid motion is damage to white matter pathways, gray matter, dura and blood vessels; disruption to the connections between and within hemispheres as well as cell death; hemorrhage becomes more common as the severity of the TBI increases. In this case, hemorrhage is seen on computer automated tomography (CAT) scans. A lower Glascow Coma Scale (GCS) score indicates greater impairment of consciousness, typically associated with a greater severity of brain injury. However, in many cases, whilst posttraumatic amnesia (PTA) often lasts for days, it does not always appear correlated with traditional CAT or magnetic resonance imaging (MRI) scans [1]. PTA does not appear to relate to the degree of diffuse axonal injury (DAI), the current most reliable marker of outcome, although very mild TBI is difficult to detect from traditional neuroimaging. For example, CAT and MRI scans can show nothing unusual in terms of shape or size of regions yet the patient may still exhibit post-concussion symptoms and reduced performance over time. Some studies have reported PTA to significantly relate to the fractional anisotropy (FA) of white matter pathways (e.g. reference [2]), with overall clinical and research findings suggesting that diffuse tensor imaging (DTI) measures may be more useful than high-resolution anatomical images in assessment of group differences [3,4]. FA is an index of white matter integrity as measured by a novel MRI technique referred to as DTI [5]; the principle underlying DTI is the hypothesis that water movement between white matter fibres is faster parallel to the fibres rather than perpendicular to them. However, there are some brain regions, as seen from traditional T1-weighted MR imaging of mild to moderate TBI patients that are clearly abnormal in shape and/or size. The most common such regions include the hippocampus (HC), inferior horns of the lateral ventricles (IHLVs), fornix, and corpus callosum (CC; e.g. [6-9], although it is the first three of these regions that will form the focus of this review.

Our hypothesis is that the combination of twisting and contrecoupe movements contributes to the nature of medial temporal lobe damage. Abnormalities in $\mathrm{HC}$ shape or size are most clearly seen from coronal and sagittal perspectives. Of course, the higher the spatial resolution the easier it is to truly appreciate these instances.

\section{The Hippocampus and Sulcal Cavities}

Hippocampal sulcal cavities (HSCs; sometimes referred to as HC Virchow Robin spaces; [10]) are small holes at the lateral aspect of the HC sulcus, representing dilatations of perivascular spaces between the cornu ammonis and dentate gyrus related to blood vessels traversing the HC sulcus [11]. Specifically, they are located next to the CA1 region which is the most vulnerable section of the HC, given its shape (that is, located along a sharp bend) and is particularly vulnerable to metabolic stress [12]. Anecdotally, HSCs are more prevalent in TBI patients cross-sectionally than in age-matched controls; HSCs are not the same as dilation of the Virchow Robin or perivascular space around cortical penetrator vessels which are more numerous in older patients and TBI patients [13]. Our review [14] found HSCs, ranging from $1 \mathrm{~mm}$ to more than $2 \mathrm{~cm}$ in length, to have an incidence across the adult life span of approximately $50 \%$; this is in close agreement with other studies of HSCs. The vestigial HC sulcus is also prominent more often in scans of TBI patients of any severity, even in the acute period, suggesting that it is not a function of age-related atrophy. Longitudinally, these HSCs become larger as does the vestigial HC sulcus (Figure 1). Hence, although HSCs have never been investigated in the context of longitudinal outcome, the degree of cognitive recovery in TBI patients is typically transient, particularly memory (which the medial temporal

*Corresponding author: Jerome J Maller, Neuroscientist, Monash Alfred Psychiatry Research Centre, The Alfred \& Monash University Central Clinical School, Level 4, 607 StKilda Rd, Melbourne 3004, Victoria, Australia, Tel: +61 3 9076 2404; Fax: +61 39076 8545; E-mail: j.maller@alfred.org.au

Received September 25, 2013; Accepted January 24, 2014; Published February 05, 2014

Citation: Maller JJ, Meslin CR (2014) Longitudinal Hippocampal and Fornix Changes after Traumatic Brain Injury: Observations from Traditional Structural Magnetic Resonance Imaging. J Neurol Neurophysiol 5: 185. doi:10.4172/21559562.1000185

Copyright: @ 2014 Maller JJ, et al. This is an open-access article distributed under the terms of the Creative Commons Attribution License, which permits unrestricted use, distribution, and reproduction in any medium, provided the original author and source are credited. 
Citation: Maller JJ, Meslin CR (2014) Longitudinal Hippocampal and Fornix Changes after Traumatic Brain Injury: Observations from Traditional Structural Magnetic Resonance Imaging. J Neurol Neurophysiol 5: 185. doi:10.4172/2155-9562.1000185

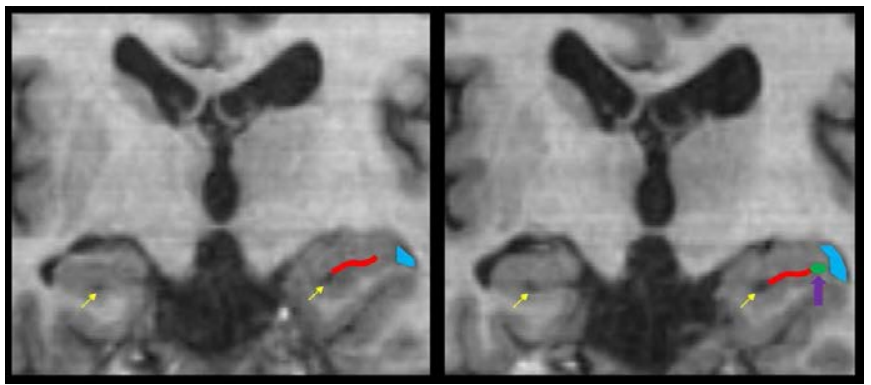

Figure 1: Hippocampal sulcal cavity (purple arrow) and vestigial hippocampal sulcus (yellow arrows and red lines) enlargement in a patient with moderate $\mathrm{TBI}$ at Time 1 (left) and Time 2 (right). Note that the lateral ventricles and inferior horn of the lateral ventricles (IHLVs, colored in blue) also become larger. The MRI scanner used was a General Electric Signa-Echospeed 1.5 Tesla (GE Healthcare, Milwaukee WI), using an eight channel head coil. The image presented was from the high-resolution $1 \mathrm{~mm}$ isotropic $\mathrm{T} 1$ weighted, three-dimensional IR prepped radio-frequency spoiled-gradient recalled-echo (3D IRSPGR) sequence (TI/TR/TE=12/300/5, TI, FA=20, slice thickness $=1 \mathrm{~mm}$ no gap, matrix $=256 \times 256$ ) with images acquired in the axial plane utilizing a $25 \mathrm{~cm}$ field of view. This pulse sequence acquires high-resolution structural data, which is the case of T1-weighted, implies a slice thickness and in-plane dimensions of no more than $1 \mathrm{~mm}$, which we have found necessary to view HSCs. Although acquired in the axial plane, the isotropic acquisition allows the data to also be viewed from other planes, such as sagittal and coronal. In our experience, the optimal plane for viewing HSCs is coronal. We have also found that HSCs are best viewed in native (raw) space as normalization may squash HSCs rendering them to no longer be visible. We urge caution when filtering as it usually blurs HSC boundaries.

lobe intimately relates to), although mild TBI is associated with subtle cognitive deficits within the first few weeks of injury that typically resolve spontaneously within 3 to 6 months $[15,16]$. HSCs are more common in TBI patients and enlarge over time at a rate greater than in the normal population, and therefore are likely to be markers of poor long-term outcome.

It is also interesting to note that the prevalence of HSCs has been related to general atrophy in both healthy and clinical populations $[14,17]$, hence it not surprising that this relationship is evident in Figure 1.

When a subject's scans are aligned so that HCs from the different time points can be superimposed, there is often a reduction of lateral and medial HC volume; this is most clearly seen from coronal view. When viewed as rendered 3-dimensional structures, the differences in these regions are more obvious (see below). These regions do not recover in volume.

\section{IHLV, Fornix and Incomplete Hippocampal Rebound}

Reduction of lateral volume in the HC head and/or body is clearly indicated when the IHLV has enlarged or appears large compared to experience with age-matched scans. Furthermore, this enlargement is commonly asymmetrical and representative of greater damage in that hemisphere compared to the other. This can be best viewed from coronal images, as demonstrated in Figure 2.

The HC sometimes appears to have been 'pushed' anteriorly, relative to age-matched scans. For example, the head of the HC in normal/healthy/control subjects always begins on, or within millimeters posteriorly to the most anterior (first) appearance of the IHLV (as seen from coronal). However, with some TBI scans the HC head begins anterior to the IHLV (Figure 3), which is essentially beneath the amygdala (AG) which traditionally is posited anteriorventral, then anterior-dorsal, and then dorsal to the $\mathrm{HC}$ head like an angled disc. This suggests it is likely that the HC was pushed forward during the TBI incident.

Considering the position of the temporal lobe, and in particular the $\mathrm{HC}$, it is vulnerable to the brunt of a closed-head impact, as demonstrated in animal TBI models (e.g. reference [18]). In a comprehensive study of structural and functional outcome after experimental TBI, Immonen et al. [19] demonstrated that the multiparametric quantitative MRI (T2, T1 $\rho$, trace of the diffusion tensor Dav, the extent of hyperintense lesion and intracerebral hemorrhage) acquired during acute and subacute phases 3 h, 3 days, 9 days and 23 days post-injury has potential to predict the functional and histopathological outcome 6 to 12 months later - specifically, the acute Dav changes in the ipsilateral HC correlated with the chronic spatial learning and memory impairment evaluated using the Morris water maze $(\mathrm{p}<0.05)$. Similarly, T1 $\rho, \mathrm{T} 2$ and $\mathrm{Dav}$ correlated with $\mathrm{HCl}$ atrophy and with histologically quantified neurodegeneration $(\mathrm{p}<0.01)$. Not surprisingly, cognitive deficits have been reported to significantly relate to compromised HC connectivity in human studies (e.g. reference [20]) and reduced HC NAA with MR spectroscopy imaging (e.g. reference [21]) and specifically to HC head damage (e.g. reference [22]). When the impact occurs, the weight of the brain shifts itself forward as it collides with the inside of the cranium; the medial temporal lobe (which the HC and AG are constituents of) is usually only a few millimeters from the temporal bone, so when the impact occurs the anterior pole of the medial temporal lobe (which contains the AG) is momentarily compressed with the weight and inertia of the brain (including the HC) behind and on top of it (hence,

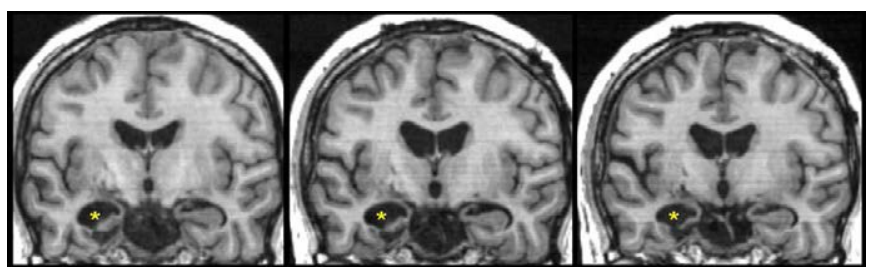

Figure 2: Reduction of lateral volume in the $\mathrm{HC}$ head and/or body in this TBI patient is clearly indicated when the IHLV (yellow asterix) has enlarged or appears large. Left=acute, Middle $=6$ months post-TBI, Right $=12$ months post$\mathrm{TBI}$. Although this represents an extreme case of atrophy for the illustration of IHLV enlargement, this was necessary to illustrate the relationship between IHLV enlargement after TBI, and time.

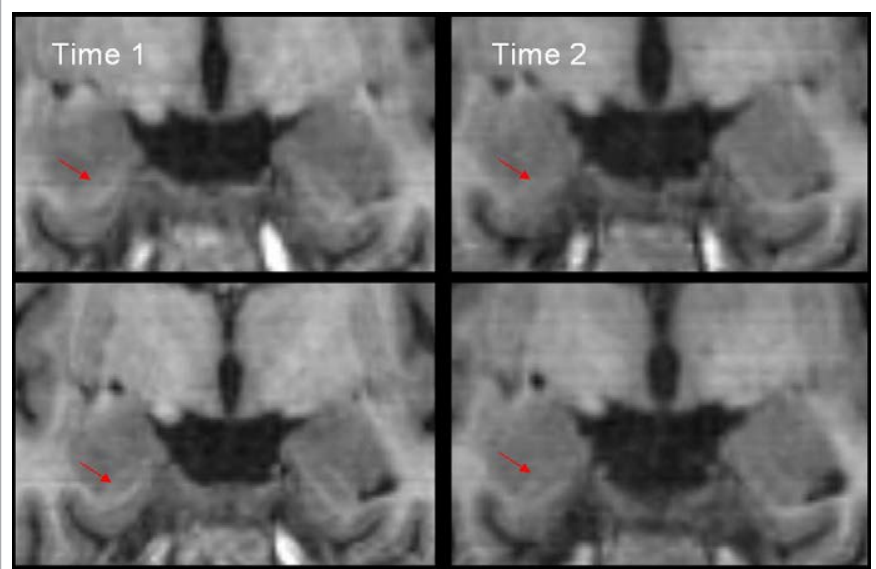

Figure 3: $\mathrm{HC}$ head can begin anterior to the IHLV. The red arrows indicate the alveus (which separates the HC from the amygdala (AG)). Top images are $7 \mathrm{~mm}$ posterior to the anterior commissure, bottom images are $8 \mathrm{~mm}$ posterior to the anterior commissure. 
the AG is stopped by hitting a wall whilst the HC continues traveling forward into the AG; the pole (including the AG) then slowly expands posteriorly with the AG expanding with it towards its original (preincident) position and size). The HC would also shift posteriorly but due to its head still being stuck in the AG, much of the HC will not return to its original position. Whilst never previously published, we describe this as "incomplete hippocampal rebound", as illustrated by the order of events in Figure 4.

The posterior portion (commonly referred to as the tail) is often the area of greatest $\mathrm{HC}$ reduction. This is not surprising given it runs along the fornix (discussed below) which commonly sustains damage after TBI. It is also in-line with prevalence of depression symptoms post-TBI given that the $\mathrm{HC}$ tail has also been implicated in major depression (e.g. reference [23]), and the fornix extends to integrate into the prefrontal cortex, a circuit which is also implicated in models of major depression [24].

When viewing scans longitudinally, the fornix sometimes appears to have shifted anteriorly. This usually occurs when the HC head has been 'pushed' forward. Hence, it is possible that the $\mathrm{HC}$ was pushed forward during the incident, and then over time the HC body and tail have 'stretched' back to a length similar to their pre-incident length, hence the fornix appears to have shifted over time but in fact because the fornix is continuous with the alveus (the layer of white matter 'covering' the $\mathrm{HC}$, and the border between the HC head and overlying AG). The position of the fornix adds reinforcement to the position of the HC; in this context the fornix may be conceptualized as holding up and stabilizing the HC from above it. Although this 'rebound' may appear to occur, the HC head does not always seem to move posteriorly as much as the rest of its divisions. An analogy may be that during the TBI incident the HC gets its head stuck in the AG but can't pull itself out when the rest of it is shifting back towards its original position pre-incident; when it does, it gets stretched. With this 'delayed stretching', the HC becomes thinner, which is in-line with observations of thinner HCs as represented by enlarged IHLVs and reduced lateral and medial gray matter described above. The rope supporting it at the back (the fornix) may be too severely damaged to pull the HC back into its original position, and during the process, the fornix becomes

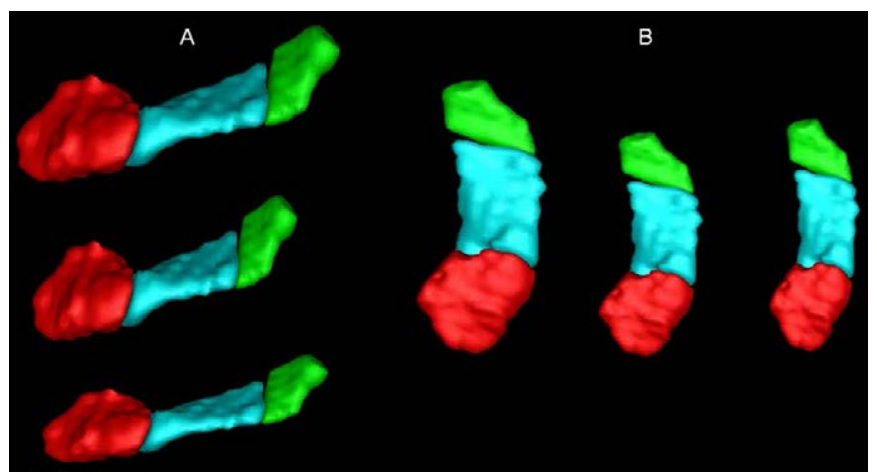

Figure 4: Temporal order of events in incomplete HC "rebound" after traumatic brain injury. Compared to a control HC (A from lateral and B from superior, top and left), the $\mathrm{HC}$ in acute period after a TBI is projected anteriorly and squashed (middle). Over the next 12 to 24 months, the HC stretches back towards its original length but never fully rebounds as it becomes thinner as represented by less medial and lateral volume (A bottom, B far right). Red=head, blue=body, green=tail. Note that the division between the tail and body, which represents the fornix, appears to shift forward and then backwards over time. Note this is a simulated series of images; that is, they have been artificially stretched in order to more clearly illustrate the $\mathrm{HC}$ rebound principle. stretched, as demonstrated by white matter hyperintensities on T2weighted imaging (e.g. reference [25]) or reduced FA (e.g. references $[26,27])$. Furthermore, this damage has been related to reduced cognitive performance. For example, Chang and colleagues [28] reported memory impairment in patients with DAI to closely related to neuronal injury of the fornix body among the three fornix regions that were assessed. Consistent with this, reduced fornix integrity (as assessed by DTI) in TBI patients has been reported to significantly correlate with reduced $\mathrm{HC}$ volume [29]. Our current research aims to investigate the relationship between HSC prevalence, HSC size, and cognition.

\section{Sensitivity, Specificity and Reliability of Detecting Abnormalities}

We have found that nearly every scan of a TBI patient has HSCs, although we have not conducted a formal study into the sensitivity, specificity, and reliability of detecting the reviewed abnormalities. Variations in source of injury are expected to affect the HC and fornix, among other structures, to different degrees. We do not have documented evidence that describes the extent of source of injury, hence we cannot comment as to how these variations in the rapid translation and/or rotation of the brain differentially impact upon different structures, although this is a focus of our current research.

Whilst there are a multitude of original reports and comprehensive reviews of morphological change of the $\mathrm{HC}$ in common disorders such as Alzheimer's disease, epilepsy, and schizophrenia [30], there are none which have reviewed this aspect of the $\mathrm{HC}$ in TBI populations. A recent review [31] found that 10 studies had collected brain MRI data in TBI patients from more than one time point and computed gross volumetric indexes (e.g. whole brain parenchymal volume, ventricleto-brain ratio), with only two of those studies having also measured $\mathrm{HC}$ volume [29,32] although only $\mathrm{Ng}$ et al. [32] presented the HC volumes (which showed reduction over time) whilst Warner and colleagues [29] only showed the HC volumes' correlations with neuropsychological performance. No analyses of HC shape were conducted.

Although HSCs are quite common in the normal population as well as clinical populations, we have observed that they are more prevalent in those who have sustained a TBI. We have not quantified the accuracy one may expect to visually detect an HSC as 'abnormal' for any diagnostic value (vs. group-level research findings), and quantifying the size of HSCs as well as their rate of atrophy would be of greater diagnostic value. We anticipate that these specific questions, that is, statistical quantification and visual quantification, will be answered by our current retrospective investigation which we have only recently begun. However, we still adhere to our main take-home message, which is that HSCs can be readily detected on T1-weighted scans with minimal image processing. Note that the 3-D renderings can be carried out with minimal training in freeware software packages after only a few clicks of the mouse.7. Conclusions

The $\mathrm{HC}$ is commonly implicated in impaired functioning in people who have sustained a traumatic brain injury. As archicortex, it is one of the oldest parts of the brain and, as such, has widespread connections with most other regions of the brain. Given the dependence of memory formation and recall upon the $\mathrm{HC}$, it plays a central role in early and late outcome. Whilst sophisticated analysis techniques are available to comprehensively elucidate specific damage and relate it to specific skill deficits, a compromised $\mathrm{HC}$ can usually be recognized from traditional 1.5T MRI without the need for reliance upon further complexity. Very recent advances in MRI sequences, such as susceptibility weighting 
Citation: Maller JJ, Meslin CR (2014) Longitudinal Hippocampal and Fornix Changes after Traumatic Brain Injury: Observations from Traditional Structural Magnetic Resonance Imaging. J Neurol Neurophysiol 5: 185. doi:10.4172/2155-9562.1000185

imaging, can reveal further information related to neuropathology which other techniques do not detect, but such methods are not yet widely available and still under development. During a process of incomplete rebound, we hypothesize that the HC shifts forwards and then backwards towards its premorbid position but thinner and less functional. The fornix is stretched and commonly damaged during a TBI, as can be seen with the naked eye from regular neuroimages. Taken together, it is clear that anatomical irregularities in the HC and fornix regions are common after a TBI and can be clearly seen on traditional neuroimages.

\section{Acknowledgements}

The authors would like to thank Professor Robin Green for use of her MRI datasets, and to Dr Richard H.S. Thomson for his assistance in preparing this manuscript.

\section{References}

1. Spitz G, Bigler ED, Abildskov T, Maller JJ, O'Sullivan R, et al. (2013) Regional cortical volume and cognitive functioning following traumatic brain injury. Brain Cogn 83: 34-44.

2. Benson RR, Meda SA, Vasudevan S, Kou Z, Govindarajan KA, et al. (2007) Global white matter analysis of diffusion tensor images is predictive of injury severity in traumatic brain injury. J Neurotrauma 24: 446-459.

3. Bendlin BB, Ries ML, Lazar M, Alexander AL, Dempsey RJ, et al. (2008) Longitudinal changes in patients with traumatic brain injury assessed with diffusion-tensor and volumetric imaging. Neuroimage 42: 503-514.

4. Maller JJ, Thomson RH, Lewis PM, Rose SE, Pannek K, et al. (2010) Traumatic brain injury, major depression, and diffusion tensor imaging: making connections. Brain Res Rev 64: 213-240.

5. Basser PJ, Mattiello J, LeBihan D (1994) MR diffusion tensor spectroscopy and imaging. Biophys J 66: 259-267.

6. Levine B, Kovacevic N, Nica El, Cheung G, Gao F, et al. (2008) The Toronto traumatic brain injury study: injury severity and quantified MRI. Neurology 70 : 771-778.

7. Strangman GE, O'Neil-Pirozzi TM, Supelana C, Goldstein R, Katz DI, et al (2010) Regional brain morphometry predicts memory rehabilitation outcome after traumatic brain injury. Front Hum Neurosci 4: 182

8. Tate DF, Bigler ED (2000) Fornix and hippocampal atrophy in traumatic brain injury. Learn Mem 7: 442-446.

9. Tomaiuolo F, Carlesimo GA, Di Paola M, Petrides M, Fera F, et al.(2004) Gross morphology and morphometric sequelae in the hippocampus, fornix and corpus callosum of patients with severe non-missile traumatic brain injury without macroscopically detectable lesions: a T1 weighted MRI study. J Neurol Neurosurg Psychiatry 75: 1314-1322.

10. Zhu YC, Dufouil C, Mazoyer B, Soumaré A, Ricolfi F, et al. (2011) Frequency and location of dilated Virchow-Robin spaces in elderly people: a populationbased 3D MR imaging study. AJNR Am J Neuroradiol 32: 709-713.

11. Sasaki M, Sone M, Ehara S, Tamakawa Y (1993) Hippocampal sulcus remnant: potential cause of change in signal intensity in the hippocampus. Radiology 188: 743-746.

12. Bartsch T, Deuschl G (2010) Transient global amnesia: functional anatomy and clinical implications. Lancet Neurol 9: 205-214.

13. Bigler ED, Maxwell WL (2011) Neuroimaging and neuropathology of TBI. NeuroRehabilitation 28: 63-74.

14. Maller JJ, Réglade-Meslin C, Chan P, Daskalakis ZJ, Thomson RH, et al.
(2011) Hippocampal sulcal cavities: prevalence, risk factors and relationship to memory impairment. Brain Res 1368: 222-230.

15. Belanger HG, Vanderploeg RD, Curtiss G, Warden DL (2007) Recent neuroimaging techniques in mild traumatic brain injury. J Neuropsychiatry Clin Neurosci 19: 5-20.

16. Bigler ED (2008) Neuropsychology and clinical neuroscience of persistent postconcussive syndrome. J Int Neuropsychol Soc 14: 1-22.

17. Maller JJ, Réglade-Meslin C, Thomson RH, Daigle M, Barr MS, et al. (2013) Hippocampal sulcal cavities in depression and healthy individuals. J Affect Disord 150: 785-789.

18. Bouilleret V, Cardamone L, Liu YR, Fang K, Myers DE, et al. (2009) Progressive brain changes on serial manganese-enhanced MRI following traumatic brain injury in the rat. J Neurotrauma 26: 1999-2013.

19. Immonen RJ, Kharatishvili I, Gröhn H, Pitkänen A, Gröhn OH (2009) Quantitative MRI predicts long-term structural and functional outcome after experimental traumatic brain injury. Neuroimage 45: 1-9.

20. Marquez de la Plata CD, Garces J, Shokri Kojori E, Grinnan J, Krishnan K, et al. (2011) Deficits in functional connectivity of hippocampal and frontal lobe circuits after traumatic axonal injury. Arch Neurol 68: 74-84

21. Capizzano AA, Jorge RE, Robinson RG (2010) Limbic metabolic abnormalities in remote traumatic brain injury and correlation with psychiatric morbidity and social functioning. J Neuropsychiatry Clin Neurosci 22: 370-377.

22. Ariza M, Serra-Grabulosa JM, Junqué C, Ramírez B, Mataró M, et al. (2006) Hippocampal head atrophy after traumatic brain injury. Neuropsychologia 44: 1956-1961.

23. Maller JJ, Daskalakis ZJ, Thomson RH, Daigle M, Barr MS, et al. (2012) Hippocampal volumetrics in treatment-resistant depression and schizophrenia: the devil's in de-tail. Hippocampus 22: 9-16.

24. Malykhin N, Concha L, Seres P, Beaulieu C, Coupland NJ (2008) Diffusion tensor imaging tractography and reliability analysis for limbic and paralimbic white matter tracts. Psychiatry Res 164: 132-142.

25. Ding K, Marquez de la Plata C, Wang JY, Mumphrey M, Moore C, et al. (2008) Cerebral atrophy after traumatic white matter injury: correlation with acute neuroimaging and outcome. J Neurotrauma 25: 1433-1440.

26. Palacios EM, Fernandez-Espejo D, Junque C, Sanchez-Carrion R, Roig T, et al. (2011) Diffusion tensor imaging differences relate to memory deficits in diffuse traumatic brain injury. BMC Neurol 11: 24.

27. Singh M, Jeong J, Hwang D, Sungkarat W, Gruen P (2010) Novel diffusion tensor imaging methodology to detect and quantify injured regions and affected brain pathways in traumatic brain injury. Magn Reson Imaging 28: 22-40.

28. Chang MC, Kim SH, Kim OL, Bai DS, Jang SH (2010) The relation between fornix injury and memory impairment in patients with diffuse axonal injury: a diffusion tensor imaging study. NeuroRehabilitation 26: 347-353.

29. Warner MA, Marquez de la Plata C, Spence J, Wang JY, Harper C, et al. (2010) Assessing spatial relationships between axonal integrity, regional brain volumes, and neuropsychological outcomes after traumatic axonal injury. J Neurotrauma 27: 2121-2130.

30. Kakeda S, Korogi Y (2010) The efficacy of a voxel-based morphometry on the analysis of imaging in schizophrenia, temporal lobe epilepsy, and Alzheimer's disease/mild cognitive impairment: a review. Neuroradiology 52: 711-721.

31. Ross DE (2011) Review of longitudinal studies of MRI brain volumetry in patients with traumatic brain injury. Brain Inj 25: 1271-1278.

32. Ng K, Mikulis DJ, Glazer J, Kabani N, Till C, et al. (2008) Magnetic resonance imaging evidence of progression of subacute brain atrophy in moderate to severe traumatic brain injury. Arch Phys Med Rehabil 89: S35-44.
This article was originally published in a special issue, Traumatic Brain Injury: Diagnosis \& Treatment handled by Editor(s). Dr. Douglas Mckay, University of Miami, USA 